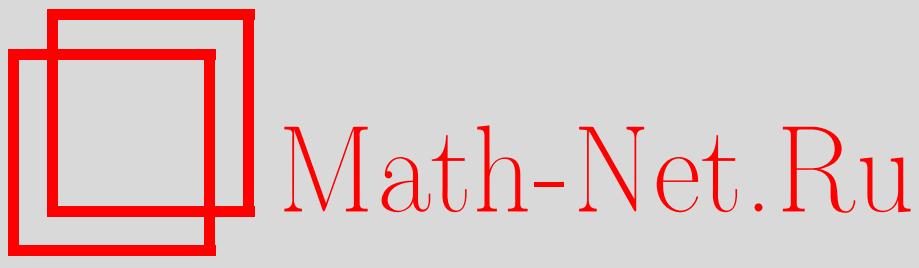

В. П. Радченко, Е. В. Небогина, М. В. Басов, Структурная модель закритического упругопластического деформирования материалов в условиях одноосного растяжения, Вестн. Сам. гос. техн. ун-та. Сер. Физ.-мат. науки, 2000, выпуск 9, 5565

DOI: https://doi.org/10.14498/vsgtu31

Использование Общероссийского математического портала Math-Net.Ru подразумевает, что вы прочитали и согласны с пользовательским соглашением

http://www.mathnet.ru/rus/agreement

Параметры загрузки:

IP : 54.172 .240 .79

26 апреля 2023 г., 12:33:54

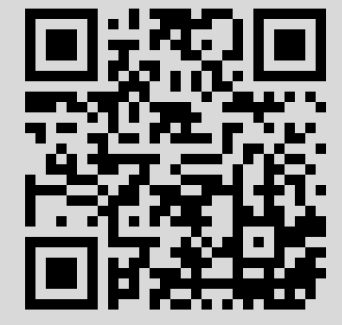




\section{СТРУКТУРНАЯ МОДЕЛЬ ЗАКРИТИЧЕСКОГО УПРУГОПЛАСТИЧЕСКОГО ДЕФОРМИРОВАНИЯ МАТЕРИАЛОВ В УСЛОВИЯХ ОДНООСНОГО РАСТЯЖЕНИЯ}

Предложена структурная модель для описания полной диаграммы упругопластического деформирования материалов при одноосном растяжении в условиях “жесткого” нагружения. С позиций механики микронеоднородных сред показана осуществимость равновесного протекания процесса накопления повреждений и разрушения материала на закритической стадии упругопластического деформирования. Проанализирована кинематика микронапряженного состояния как на стадии упрочнения, так и на стадии разрушения материала. Выполнена обстоятельная экспериментальная проверка структурной модели. Наблюдается хорошее соответствие расчетных и экспериментальных диаграмм упругопластического деформирования.

Потеря несущей способности одноосного образца при растяжении с феноменологических позиций является результатом накопления повреждений в результате пластического деформирования, сопровождающегося необратимым изменением внутренней структуры материала. Вопрос описания полной диаграммы упругопластического деформирования достаточно сложной и до настоящего времени во всей его полноте не раскрыт. Основная проблема заключается в объяснении и описании ниспадающего участка диаграммы, так называемой стадии неустойчивого (закритического) деформирования [1,2]. Факторов, влияющих на вид диаграммы, достаточно много.

В [3] отмечалось, что сопротивление разрушению является не только свойством материала, но и зависит от жесткости нагружающей системы, в которую входят как нагружающее устройство, так и само деформируемое тело, окружающее зону разрушения. Существенно сказывается на характере ниспадающей ветви диаграммы и режим нагружения. Так, когда к находящемуся в однородном напряженном состоянии телу прикладываются не зависящие от его сопротивления силы, разрушению соответствуют максимально достижимые значения напряжений, как это обычно и принимается в расчетах на прочность. В другом же предельном случае «жесткого нагружения» (когда задаются перемещения точек на границе) возможно равновесное протекание процесса накопления повреждений, что находит свое отражение на ниспадающей ветви диаграммы деформирования. С аналогичных позиций это явление описывалось и в ряде других работ [4-6].

Наличие ниспадающей ветви на диаграмме деформирования привело к тому, что характеристикой разрушения материала стали считать не точку локального экстремума зависимости «перемещение-нагрузка», а конечную точку диаграммы. Фридман Я.Б. [7] отмечает, что эта точка отражает состояние, соответствующее началу заключительной быстропротекающей неравновесной стадии процесса разрушения, при этом разрушение заканчивается при усилии, близком к нулю.

Вообще говоря, в вопросе возможности построения полной диаграммы существуют диаметрально противоположные мнения. Так, Г.А. Черепанов считает [8], что закритический участок является динамической характеристикой системы образец- испытательная машина в целом и «вообще не зависит от физических свойств материала в закритической области». С другой стороны, в работах $[9,10]$ теоретически обосновывается осуществимость состояний материала, соответствующих ниспадающей ветви диаграммы деформирования, без учета эффекта накопления повреждений.

В свою очередь, ряд экспериментальных работ А.А. Лебедева с соавторами [11-13] показал, что ниспадающая ветвь все же зависит не только от жесткости испытательной машины, но и является характеристикой материала, при этом независимо от класса и исходной структуры материала кинетика разгружения носит общий стадийный характер. Наличие ниспадающего участка диаграммы свидетельствует о том, что разрушение материала не является мгновенным актом, а проистекает непрерывно в течение определенного времени. На это же указывалось и В.В. Стружановым, и В.И. Мироновым [14]. Исследование кинетики разрушения образцов при растяжении показали, что сначала происходит разрыхление материала, образуются микропоры и микротрещины, т.е. происходит рассеянное накопление поврежденности в материале на уровне микроструктуры [15]. Этот процесс можно рассматривать как деформационное разу- 
прочнение материала. Затем возникает магистральная внутренняя трещина, и с этого момента резко меняется сопротивление образца, и нагрузка начинает падать.

Таким образом, по всей видимости, падение сопротивления в ходе равновесного необратимого пластического деформирования является следствием уменьшения эффективной площади сечения элементарного объема материала, воспринимающего нагрузку. Теоретические истоки описания этого экспериментального факта заложены еще в работах Ю.Н. Работнова [16], И.И. Новожилова [17], Л.М. Качанова [18].

Целью настоящей работы является разработка математической модели микронеоднородной среды, которая бы описывала полную диаграмму упругопластического деформирования, включая участок закритического деформирования, в случае «жесткого» нагружения. Для этого используется структурная модель среды стержневого типа, предложенная в [19,20].

Хорошо известно, что конструкционные металлические и природные материалы даже малого объема с точки зрения механики микронеоднородных сред представляют сложную статически неопределимую систему случайно ориентированных кристаллических зерен. Поэтому, исходя из общих принципов построения структурных математических моделей микронеоднородных сред [21,22], в настоящей работе поликристаллический материал моделируется системой хаотически ориентированных однородных стержней (локальных элементов) одинаковой длины, работающих на растяжение-сжатие. Каждый локальный элемент этой системы наделяется простейшими деформационными свойствами: линейной упругостью и идеальной пластичностью, которые, по-видимому, являются основными микромеханизмами упругопластической деформации [23-25]. В таком случае задача одноосного деформирования образца сводится к

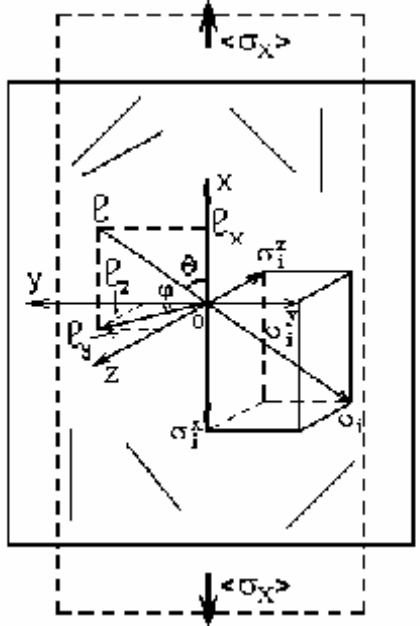

Р и с. 1.Схематическое изображение структурной модели изучению поведения стержневой конструкции (рис.1), а деформация каждого локального элемента системы представляется в виде

$$
\varepsilon_{i}=e_{i}+e_{i}^{p},
$$

где $e_{i}=\sigma / E_{M}-$ упругая деформация $\left(E_{M}-\right.$ микромодуль Юнга); $e_{i}^{p}$ - микропластическая деформация, подчиняющаяся закону идеальной пластичности; $\sigma_{i}$ - микронапряжение в локальном элементе; $i$ - номер стержня. Ориентация локального элемента задается углами $\theta$ и $\varphi$ (см. рис.1), где $\Theta$-угол между локальным элементом и осью $\mathrm{OX}, \varphi-$ угол между проекцией стержня на плоскость OYZ и осью OY $(0 \leq \Theta \pi / 2 ; 0 \leq \varphi \leq 2 \pi)$.

Обозначим через $\sigma=\sigma(\Theta, \varphi)$ напряжение, возникающее в локальном элементе (микронапряжение); $\varepsilon=\varepsilon(\Theta, \varphi)$ деформацию локального элемента (микродеформацию); $<\sigma_{x}>$ - микронапряжение, приложенное к образцу; $<\varepsilon_{x}>$,

$<\varepsilon_{y}>,\left\langle\varepsilon_{z}>\right.$ - продольную и поперечные микродеформации образца соответственно. В силу симметрии задачи все характеристики микронапряженного состояния будут зависеть только от угла $\Theta$, при этом $<\varepsilon_{y}>=<\varepsilon_{z}>$.

В $[19,20]$ для рассматриваемой структурной модели были получены уравнения равновесия

$$
\left.\begin{array}{l}
<\sigma_{x}>=2 \int_{0}^{\frac{\pi}{2}} \sigma\left(\Theta \cos ^{2} \Theta \sin \Theta d \Theta\right. \\
\int_{0}^{\frac{\pi}{2}} \sigma(\Theta) \sin ^{3} \Theta d \Theta=0
\end{array}\right\}
$$

и уравнения совместности деформаций

$$
\varepsilon(\Theta)=<\varepsilon_{x}>\cos ^{2} \Theta+<\varepsilon_{y}>\sin ^{2} \Theta .
$$

Для установления связи между микро- и макродеформацией введена гипотеза однородности деформации по объему в виде 


$$
<\varepsilon_{x}>=\varepsilon(0),<\varepsilon_{y}>=<\varepsilon_{z}>=\varepsilon\left(\frac{\pi}{2}\right) .
$$

Для соответствующего расчета диаграммы упругопластического деформирования на основании структурной модели необходимо знать величину микропредела текучести локального элемента $\sigma_{T M}$ и величину микромодуля Юнга $E_{M}$. В $[19,20]$ были установлены следующие зависимости для их вычисления:

$$
\sigma_{T M}=3 \sigma_{\Pi P}, E_{M}=3<E>
$$

где $\sigma_{\Pi P}$ - предел пропорциональности (упругости) на диаграмме упругопластического деформирования образца, $\langle E\rangle$ - макромодуль Юнга.

Если стандартная схематическая диаграмма упругопластического макродеформирования образца имеет вид, представленный на рис. 2 , то кинетика поля микронапряжений в процессе упругопластического деформирования и разрушения металлов, изображенная на рис. 3 , состоит из этапов $a-e$ (см. рис.3).

Точке 1 на диаграмме деформирования (см. рис.2) соответствует чисто упругое состояние, поэтому для эпюры микронапряжений имеем $|\sigma(\Theta)|<\sigma_{T M}, 0 \leq \Theta \leq \pi / 2$ (случай $a$ на рис. 3)

По мере возрастания нагрузки (точка 2 на рис.2) часть локальных элементов модели достигает предела текучести и возникает зона пластического растяжения $\left(\sigma(\Theta)=\sigma_{T M}, 0 \leq \Theta \leq \alpha_{1}\right)$, остальные элементы находятся в упругом состоянии $\left.(\sigma(\theta))<\sigma_{T M}, \alpha_{1}<\theta \leq \pi / 2\right)$. Этому состоянию соответствует схема $\sigma$ на рис. 3. Дальнейшее увеличение нагрузки приводит к увеличению зоны пластически растяну-

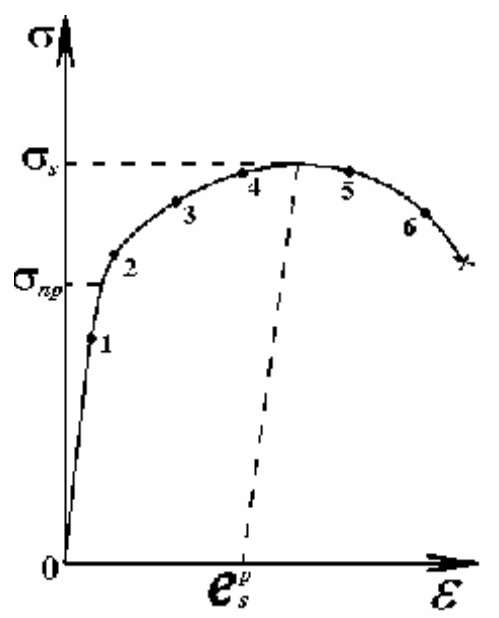

Рис. 2. Диаграмма упругопластического деформирования образца тых локальных элементов модели и росту (по модулю) микронапряжений сжатия до тех пор, пока напряжение в стержне при $\Theta=\pi / 2$ не достигнет микропредела при сжатии: $\sigma(\pi / 2)=-\sigma_{T M}$ ( схема в на рис.3 ). Начиная с этого момента в материале образуются зоны пластического растяжения $\left(0 \leq \Theta \leq \alpha_{1}\right)$, зоны пластического сжатия $\left(\alpha_{1} \leq \Theta \leq \pi / 2\right)$ и упругая зона $\left(\alpha_{1} \leq \Theta \leq \alpha_{2}\right)$. Этому состоянию соответствует схема 2 на рис. 3. Очевидно, что схема 2 реализуется до состояния, соответствующего на диаграмме упругопластического деформирования временному пределу сопротивления $\sigma_{s}$ (максимум зависимости $\sigma=\sigma(\varepsilon)$, рис. 2 ). В последующем на закритической стадии деформирования естественно предположить, что наиболее нагруженные локальные элементы модели в зоне растяжения начинают разрушаться и микронапряженное состояние среды определяется зоной разрушенных элементов $0 \leq \theta \leq \alpha_{3}$, зонами пластического растяжения $\alpha_{3} \leq \theta \leq \alpha_{1}$ и пластического сжатия $\alpha_{1} \leq \theta \leq \pi / 2$, упругой областью $\alpha_{1} \leq \theta \leq \alpha_{2}$ (схема $\partial$ на рис.3). Завершающей стадии закритического деформирования соответствуют зоны разрушения в областях пластического растяжения ( $\left.0 \leq \Theta \leq \alpha_{3}\right)$ и пластического сжатия $\left(\alpha_{4} \leq \Theta \leq \pi / 2\right)$, пластического сжатия $\left(\alpha_{4} \leq \Theta \leq \pi / 2\right)$ и пластического растяжения $\left(\alpha_{3} \leq \Theta \leq \alpha_{1}\right)$ без разрушения локальных элементов, а также упругая область $\left(\alpha_{1} \leq \Theta \leq \alpha_{2}\right)$. Схематически это состояние представлено схемой $e$ на рис.3.

Вопрос, связанный с выбором критериев разрушения локального элемента, будет рассмотрен ниже при математическом анализе соответствующих схем.

Рассмотрим теперь вопрос построения математических моделей для случаев $a$ - $e$, представленных на рис.3.

a. Диаграмма микронапряжений в данном случае соответствует упругому состоянию. Исходя из уравнений (1)-(3) и закона Гука нетрудно установить, что

$$
\sigma(\theta)=3\left\langle\sigma_{x}\right\rangle\left(\cos ^{2} \theta-\frac{1}{4} \sin ^{2} \theta\right) \text {. }
$$

Из соотношения (5) следует, что при одноосном растяжении микронапряжения в упругой области находятся в пределах 


$$
3<\sigma_{x}>\leq \sigma(\theta) \leq-\frac{3}{4}<\sigma_{x}>,
$$

т. е. при одноосном растяжении в образце возникают значительные сжимающие микронапряжения, за счет которых и возникают поперечные макродеформации.
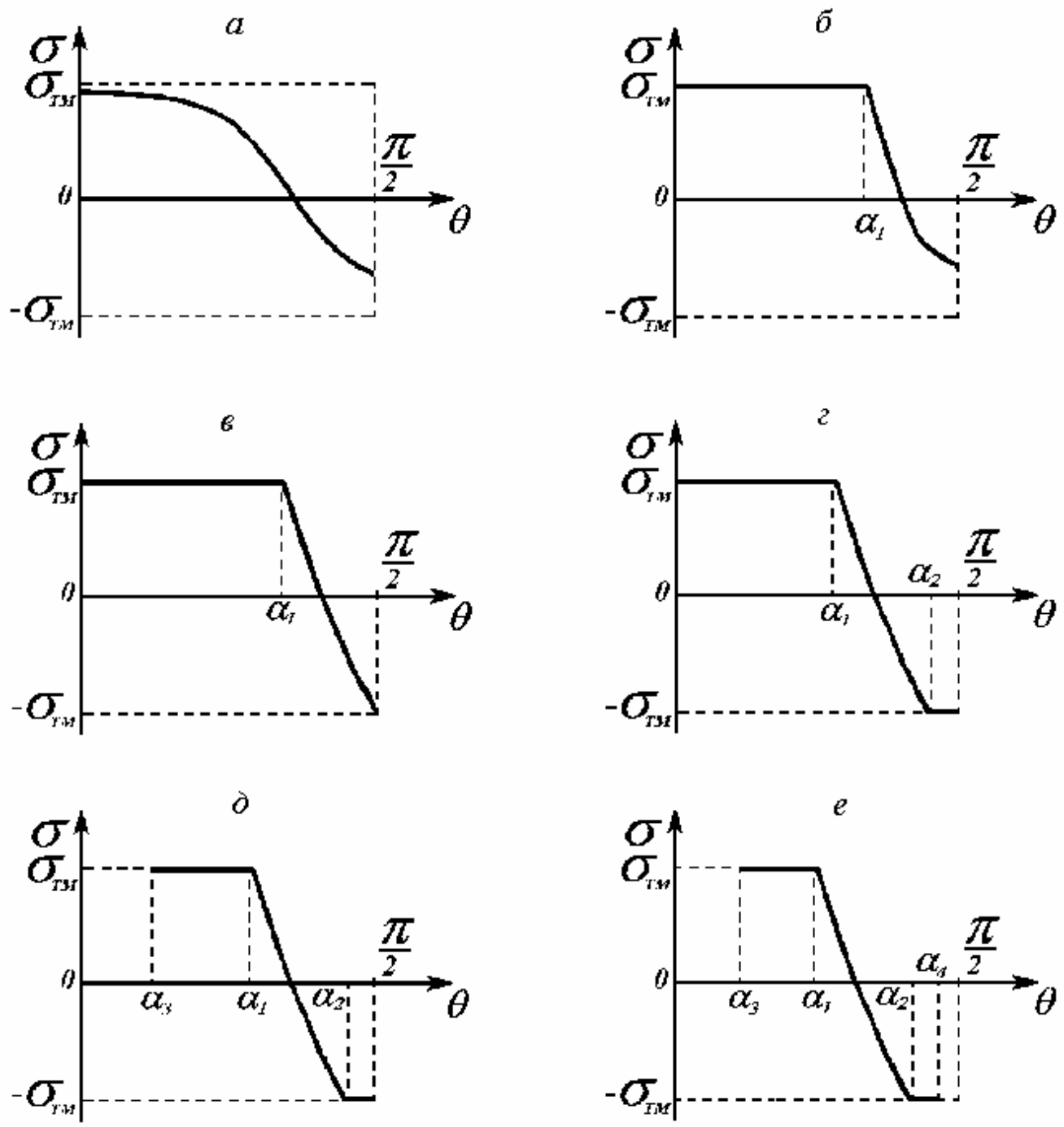

Р и с. 3. Диаграммы распределения микронапряжений в процессе упругопластического деформирования

б. В этом случае часть стержней находится в состоянии упругопластического растяжения, т. е. $\sigma(\Theta)=\sigma_{T M}$ при $0 \leq \Theta \leq \alpha_{l}$, но разрушения ни одного локального элемента не наблюдается. Область же $\alpha_{1} \leq \theta \leq \pi / 2$ соответствует чисто упругому состоянию локальных элементов.

Опишем математически данную схему. Из уравнения совместности (2) при $\theta=\alpha_{1}$ имеем

$$
\varepsilon\left(\alpha_{1}\right)=<\varepsilon_{x}>\cos ^{2} \alpha_{1}+<\varepsilon_{y}>\sin ^{2} \alpha_{1} .
$$

Поскольку граничный между пластической и упругой областями элемент при $\Theta=\alpha_{1}$ находится в упругом состоянии, также как и элемент при $\Theta=\pi / 2$, то с учетом (3) и закона Гука для локального элемента соотношение (6) примет вид

$$
\frac{\sigma_{T M}}{E_{M}}=<\varepsilon_{x}>\cos ^{2} \alpha_{1}+\frac{\sigma\left(\frac{\pi}{2}\right)}{E_{M}} \sin ^{2} \alpha_{1},
$$

откуда

$$
<\varepsilon_{x}>=\frac{1}{E_{M} \cos ^{2} \alpha_{1}}\left(\sigma_{T M}-\sigma\left(\frac{\pi}{2}\right) \sin ^{2} \alpha_{1}\right)
$$


Подставляя (7) в (2) и используя закон Гука, в упругой области для $\alpha_{1} \leq \Theta \leq \pi / 2$ получим

$$
\sigma(\Theta)=\frac{1}{\cos ^{2} \alpha_{1}}\left[\sigma_{T M}-\sigma\left(\frac{\pi}{2}\right) \sin ^{2} \alpha_{1}\right] \cos ^{2} \Theta+\sigma\left(\frac{\pi}{2}\right) \sin ^{2} \Theta .
$$

Запишем соотношение (1) в виде аддитивных составляющих по упругопластической и упругой областям:

$$
\begin{gathered}
<\sigma_{x}>=2 \int_{0}^{\alpha_{1}} \sigma_{T M} \cos ^{2} \Theta \sin \Theta d \Theta+\int_{\alpha_{1}}^{\pi / 2} \sigma(\theta) \cos ^{2} \theta \sin \Theta d \Theta ; \\
\int_{0}^{\alpha_{1}} \sigma_{T M} \sin ^{3} \Theta d \Theta+\int_{\alpha_{1}}^{\pi / 2} \sigma(\Theta) \sin ^{3} \Theta d \Theta=0 .
\end{gathered}
$$

Подставляя (8) в (9) и интегрируя полученное, имеем следующую систему уравнений:

$$
\left\{\begin{array}{l}
<\sigma_{x}>=\frac{2}{15}\left[5 \sigma_{T M}-2 \cos ^{3} \alpha_{1}\left(\sigma_{T M}-\sigma\left(\frac{\pi}{2}\right)\right)\right] \\
5 \sigma_{T M}-5 \cos \alpha_{1}\left(\sigma_{T M}-\sigma\left(\frac{\pi}{2}\right)+\cos ^{3} \alpha_{1}\left(\sigma_{T M}-\sigma\left(\frac{\pi}{2}\right)\right)\right)=0 .
\end{array}\right.
$$

Неизвестными в (10) являются $\left\langle\sigma_{x}>, \alpha_{1}, \sigma(\pi / 2)\right.$, т.е. имеем три неизвестных и два уравнения. Однако в процессе упругопластического нагружения при феноменологическом подходе известен некоторый параметр нагружения. В настоящей работе рассматривается случай «жесткого» нагружения, т. е. считается известной величина $\left\langle\dot{\varepsilon}_{x}>\right.$, а значит, и $\left\langle\varepsilon_{x}>\right.$, которая однозначно связана с величиной $\alpha_{1}$ соотношением (7). Поэтому в (10) одну из величин $\alpha_{1}$ или $<\varepsilon_{x}>$ можно задавать, считая ее параметром нагружения, и решать систему (10) относительно двух других неизвестных.

Положим в качестве известной величину $\alpha_{1}$. Тогда задавая значение величины $\alpha_{1}$, из второго уравнения системы (10) находим $\sigma(\pi / 2)$, затем из первого уравнения - $\left\langle\sigma_{x}\right\rangle$, а из (7) $<\varepsilon_{x}>$.

в. Для схемы, представленной на рис.3, в, имеем ,что $\sigma(\theta)=\sigma_{T M}\left(0 \leq \theta \leq \alpha_{1}\right)$, $\sigma\left(\frac{\pi}{2}\right)=-\sigma_{T M}$, а локальные элементы, соответствующие углам $\alpha_{1} \leq \theta<\pi / 2$, находятся в упругом состоянии. Аналогично рассмотренному выше случаю, подставляя значения микронапряжений в уравнения равновесия (1), используя уравнение совместности (2) и закон Гука, получим

$$
\left\{\begin{array}{l}
<\sigma_{x}>=\frac{2 \sigma_{T M}\left(5-4 \cos ^{3} \alpha_{1}\right)}{15} \\
5+2 \cos ^{3} \alpha_{1}-10 \cos \alpha_{1}=0 \\
<\varepsilon_{x}>=\frac{\sigma_{T M}}{15}\left(1+\sin ^{2} \alpha_{1}\right) .
\end{array}\right.
$$

Из второго уравнения определяется значение $\alpha_{1}$, что позволяет найти $\left\langle\sigma_{x}>\right.$ и $\left\langle\varepsilon_{x}>\right.$. В частности, распределение поля микронапряжений в упругой области $\alpha_{1}<\theta<\pi / 2$ имеет вид

$$
\sigma(\theta)=\frac{\sigma_{T M}}{\cos ^{2} \alpha_{1}}\left(\cos ^{2} \theta+\sin ^{2} \alpha_{1} \cos ^{2} \theta-\sin ^{2} \theta \cos ^{2} \alpha_{1}\right)
$$

2. Для данного состояния материала (рис. 3, г) при $0<\theta<\alpha_{1}$ имеем $\sigma(\theta)=\sigma_{T M}$; при $\alpha_{2} \leq \theta \leq \pi / 2-\sigma(\theta)=-\sigma_{T M}$; а в области $\alpha_{1} \leq \theta \leq \alpha_{2}$ будет упругое распределение микронапряжений. Предполагается, что в этой области разрушения локальных элементов также не происходит.

Запишем уравнения совместимости (2) для углов $\theta=\alpha_{1}$ и $\theta=\alpha_{2}$ с учетом закона Гука: 


$$
\left\{\begin{array}{l}
\frac{\sigma\left(\alpha_{1}\right)}{E_{M}}=<\varepsilon_{x}>\cos ^{2} \alpha_{1}+<\varepsilon_{y}>\sin ^{2} \alpha_{1}, \\
\frac{\sigma\left(\alpha_{2}\right)}{E_{M}}=<\varepsilon_{x}>\cos ^{2} \alpha_{2}+<\varepsilon_{y}>\sin ^{2} \alpha_{2} .
\end{array}\right.
$$

Решая систему (12) относительно $<\varepsilon_{x}>$ и $\left\langle\varepsilon_{y}>\right.$, с учетом $\sigma\left(\alpha_{1}\right)=\sigma_{\text {тм }}, \sigma\left(\alpha_{2}\right)=-\sigma_{\text {тм }}$, получим

$$
<\varepsilon_{x}>=-\frac{\sigma_{\mathrm{TM}}}{\mathrm{E}_{\mathrm{M}}} \frac{\sin ^{2} \alpha_{1}+\sin ^{2} \alpha_{2}}{\cos ^{2} \alpha_{2}-\cos ^{2} \alpha_{1}} ;<\varepsilon_{y}>=\frac{\sigma_{\mathrm{TM}}}{\mathrm{E}_{\mathrm{M}}} \frac{\cos ^{2} \alpha_{1}+\cos ^{2} \alpha_{2}}{\cos ^{2} \alpha_{2}-\cos ^{2} \alpha_{1}} .
$$

В упругой области для $\alpha_{1} \leq \Theta \leq \alpha_{2}$ из (2) имеем

$$
\sigma(\Theta)=\mathrm{E}_{\mathrm{M}}\left(<\varepsilon_{x}>\cos ^{2} \Theta+<\varepsilon_{y}>\sin ^{2} \Theta\right) .
$$

Подставляя в (14) соотношения (13), находим упругое распределение микронапряжений $\left(\alpha_{1} \leq \Theta \leq \alpha_{2}\right)$ :

$$
\sigma(\Theta)=\sigma_{\text {TM }}\left[-\frac{\sin ^{2} \alpha_{1}+\sin ^{2} \alpha_{2}}{\cos ^{2} \alpha_{2}-\cos ^{2} \alpha_{1}} \cos ^{2} \Theta+\frac{\cos ^{2} \alpha_{1}+\cos ^{2} \alpha_{2}}{\cos ^{2} \alpha_{2}-\cos ^{2} \alpha_{1}} \sin ^{2} \Theta\right] .
$$

Запишем теперь уравнения равновесия (1):

$$
\begin{aligned}
& <\sigma_{x}>=2\left[\int_{0}^{\alpha_{1}} \sigma_{\mathrm{TM}} \cos ^{2} \Theta \sin \Theta d \Theta+\int_{\alpha_{1}}^{\alpha_{2}} \sigma(\Theta) \cos ^{2} \Theta \sin \Theta d \Theta-\int_{\alpha_{2}}^{\frac{\pi}{2}} \sigma_{\mathrm{TM}} \cos ^{2} \Theta \sin \Theta d \Theta\right] ; \\
& \int_{0}^{\alpha_{1}} \sigma_{\mathrm{TM}} \sin ^{3} \Theta d \Theta+\int_{\alpha_{1}}^{\alpha_{2}} \sigma(\Theta) \sin ^{3} \Theta d \Theta-\int_{\alpha_{2}}^{\frac{\pi}{2}} \sigma_{\mathrm{TM}} \sin ^{3} \Theta d \Theta=0,
\end{aligned}
$$

где $\sigma(\Theta)$ задается (15). Интегрируя (16), получим

$$
\left\{\begin{array}{l}
\left.<\sigma_{x}\right\rangle=\frac{2 \sigma_{\mathrm{TM}}}{15\left(\cos ^{2} \alpha_{2}-\cos ^{2} \alpha_{1}\right)} \cdot\left[5\left(\cos ^{2} \alpha_{2}-\cos ^{2} \alpha_{1}\right)-4\left(\cos ^{5} \alpha_{2}-\cos ^{5} \alpha_{1}\right)\right] \\
2\left(\cos ^{5} \alpha_{2}-\cos ^{5} \alpha_{1}\right)-10\left(\cos ^{3} \alpha_{2}-\cos ^{3} \alpha_{1}\right)+5\left(\cos ^{2} \alpha_{2}-\cos ^{2} \alpha_{1}\right)=0 .
\end{array}\right.
$$

Полная система уравнений для рассматриваемого случая состоит из уравнений (13) и (17). Неизвестными в этой системе являются $\alpha_{1}, \alpha_{2},\left\langle\sigma_{\mathrm{x}}\right\rangle,\left\langle\varepsilon_{\mathrm{x}}\right\rangle,\left\langle\varepsilon_{\mathrm{y}}\right\rangle$, т. е. пять неизвестных, а уравнений - четыре. Но по вышеизложенным соображениям одну из неизвестных можно считать параметром нагружения и задавать, а далее решать систему четырех уравнений относительно остальных четырех неизвестных. Поэтому алгоритм решения системы (13), (17) может быть следующим: задавая значение угла $\alpha_{2}<\frac{\pi}{2}$, из второго уравнения (17) определяется значение угла $\alpha_{1}$, а затем последовательно определяются $\left\langle\sigma_{\mathrm{x}}\right\rangle,\left\langle\varepsilon_{\mathrm{x}}\right\rangle,\left\langle\varepsilon_{\mathrm{y}}\right\rangle$.

$\partial$. Перейдем теперь к описанию следующего этапа деформирования материала, сопровождающегося разрушением локальных элементов модели (рис.3, $\partial$ ). Но для этого необходимо ввести критерий разрушения локального элемента системы.

Будем использовать энергетический подход, предполагая, что разрушение локального элемента наступает тогда, когда работа микронапряжения на микродеформации пластичности достигает критического значения. В качестве критического будем использовать состояние материала, соответствующее локальному экстремуму на диаграмме упругопластического макродеформирования образца, характеризующегося точкой $\left(\sigma_{S}, e_{S}^{p}\right)$ (см. рис. 2) и отделяющего участки устойчивого и неустойчивого (закритического) деформирования. Тогда элементарный стержень при $\Theta=$ const находится в неразрушенном состоянии, если

$$
\int_{0}^{e^{p}} \sigma(\Theta) d e^{p}(\Theta)<A_{p}^{*}
$$

и разрушается, если выполняется условие 


$$
\int_{0}^{e^{p}} \sigma(\Theta) d e^{p}(\Theta)=A_{p}^{*}
$$

где $A_{p}^{*}$ - критическая величина работы (характеристика материала).

В силу того, что в пластическом состоянии в зоне растяжения $\sigma(\Theta)=\sigma_{\text {тм }}$ и $e^{p}(\Theta)>0$, а в зоне сжатия $\sigma(\Theta)=-\sigma_{\text {тм }}$ и $e^{p}(\Theta)<0$, неравенство (18) принимает вид

$$
\sigma_{\mathrm{TM}}\left|e^{p}(\Theta)\right|<A_{p}^{*} .
$$

Величину $A_{p}^{*}$ можно определить следующим образом. Первым разрушится наиболее нагруженный локальный элемент при $\Theta=0$. Поскольку в силу гипотезы однородности деформации по объему (3) в момент разрушения имеем, что $e^{p}(0)=<\varepsilon_{x}>=e_{S}^{p}$, то тогда из (19) получим

$$
A_{p}^{*}=\int_{0}^{e_{S}^{p}} \sigma_{\mathrm{TM}} d e^{p}(0)=\sigma_{\mathrm{TM}} e_{S}^{p}
$$

С учетом (21) критерий разрушения (19) принимает деформационный характер и имеет вид

$$
\left|e^{p}(\Theta)\right|=e_{S}^{p} .
$$

Учитывая вышеизложенное, рассмотрим схему деформирования, представленную на pис. 3, $\partial$. Здесь при $0 \leq \Theta \leq \alpha_{3}$ локальные элементы модели разрушены; при $\alpha_{3} \leq \Theta \leq \alpha_{1}$ имеем $\sigma(\Theta)=\sigma_{\mathrm{TM}}$; при $\alpha_{1} \leq \Theta \leq \alpha_{2}$ будет упругое состояние, а при $\alpha_{2} \leq \Theta \leq \pi / 2-\sigma(\Theta)=-\sigma_{T M}$.

Записывая уравнения совместности деформаций (2) для $\Theta=\alpha_{1}, \Theta=\alpha_{2}$ с учетом закона Гука, получим систему (12), решение которой относительно $\left\langle\varepsilon_{\mathrm{x}}\right\rangle,<\varepsilon_{\mathrm{y}}>$ имеет вид (13).

При $\Theta=\alpha_{3}$ из (2) ( с учетом выражений для $<\varepsilon_{\mathrm{x}}>,<\varepsilon_{\mathrm{y}}>$ из (13)) имеем

$$
\frac{\sigma_{\mathrm{TM}}}{\mathrm{E}_{\mathrm{M}}}+e_{S}^{p}=-\frac{\sigma_{\mathrm{TM}}}{\mathrm{E}_{\mathrm{M}}}\left[\frac{\sin ^{2} \alpha_{1}+\sin ^{2} \alpha_{2}}{\cos ^{2} \alpha_{2}-\cos ^{2} \alpha_{1}} \cos ^{2} \alpha_{3}-\frac{\cos ^{2} \alpha_{1}+\cos ^{2} \alpha_{2}}{\cos ^{2} \alpha_{2}-\cos ^{2} \alpha_{1}} \sin ^{2} \alpha_{3}\right] \text {. }
$$

Записывая уравнения равновесия (1), получим

$$
\begin{aligned}
& <\sigma_{x}>=2\left[\int_{\alpha_{3}}^{\alpha_{1}} \sigma_{\mathrm{TM}} \cos ^{2} \Theta \sin \Theta d \Theta+\int_{\alpha_{1}}^{\alpha_{2}} \sigma(\Theta) \cos ^{2} \Theta \sin \Theta d \Theta-\int_{\alpha_{2}}^{\frac{\pi}{2}} \sigma_{\mathrm{TM}} \cos ^{2} \Theta \sin \Theta d \Theta\right] ; \\
& \int_{\alpha_{3}}^{\alpha_{1}} \sigma_{\mathrm{TM}} \sin ^{3} \Theta d \Theta+\int_{\alpha_{1}}^{\alpha_{2}} \sigma(\Theta) \sin ^{3} \Theta d \Theta-\int_{\alpha_{2}}^{\frac{\pi}{2}} \sigma_{\mathrm{TM}} \sin ^{3} \Theta d \Theta=0,
\end{aligned}
$$

где $\sigma(\Theta)$ в упругой области задается так же, как и в случае г соотношением (15). Интегрируя (24) и присоединяя к полученному (13), (23) имеем следующую систему уравнений:

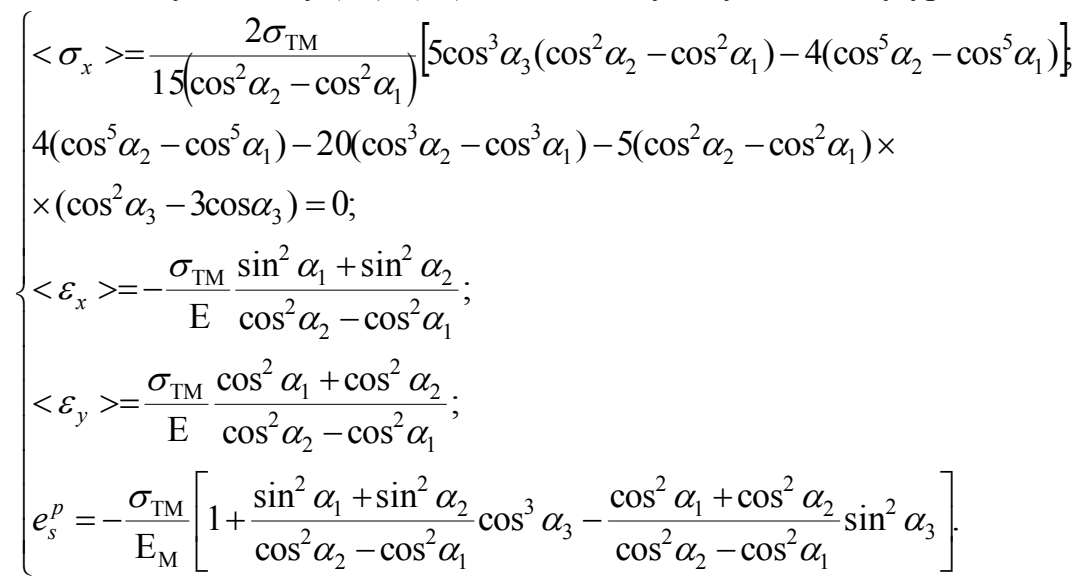

Алгоритм решения системы (25) может быть следующим: задается значение угла $\alpha_{3}$, играющего роль параметра нагружения; из системы уравнений, образованной вторым и пятым 
уравнениями (25), определяются значения $\alpha_{1}$ и $\alpha_{2}$; зная $\alpha_{1}, \alpha_{2}, \alpha_{3}$. находим последовательно $<\varepsilon_{\mathrm{x}}>,<\varepsilon_{\mathrm{y}}>,<\sigma_{\mathrm{x}}>$.

e. Рассмотрим заключительный этап разрушения образца, изображенный схематически на рис. 3, е. Здесь разрушаются не только элементы в зоне пластического растяжения при $0 \leq \Theta \leq \alpha_{3}$, но и в зоне пластического сжатия $\alpha_{4} \leq \Theta \leq \pi / 2$. В области $\alpha_{3} \leq \Theta \leq \alpha_{1}$ имеем $\sigma(\Theta)=\sigma_{T M}$; при $\alpha_{4} \leq \Theta \leq \alpha_{2}-\sigma(\Theta)=-\sigma_{T M}$, а в зоне $\alpha_{1} \leq \Theta \leq \alpha_{2}$ локальные элементы находятся в упругой области.

Записывая уравнения совместности деформации для $\Theta=\alpha_{1}, \Theta=\alpha_{2}, \Theta=\alpha_{3}, \Theta=\alpha_{4}$, подставляя напряжение $\sigma(\Theta)$ в уравнения равновесия и выполняя их интегрирование, получим следующую математическую модель для рассматриваемого случая:

$$
\left\{\begin{array}{l}
<\varepsilon_{x}>=-\frac{\sigma_{\mathrm{TM}}}{\mathrm{E}} \frac{\sin ^{2} \alpha_{1}+\sin ^{2} \alpha_{2}}{\cos ^{2} \alpha_{2}-\cos ^{2} \alpha_{1}} \\
<\varepsilon_{y}>=\frac{\sigma_{\mathrm{TM}}}{\mathrm{E}} \frac{\cos ^{2} \alpha_{1}+\cos ^{2} \alpha_{2}}{\cos ^{2} \alpha_{2}-\cos ^{2} \alpha_{1}} \\
\frac{\sigma_{\mathrm{TM}}}{\mathrm{E}_{\mathrm{M}}}+e_{s}^{p}=<\varepsilon_{x}>\cos ^{2} \alpha_{3}+<\varepsilon_{y}>\sin ^{2} \alpha_{3} \\
-\frac{\sigma_{\mathrm{TM}}}{\mathrm{E}_{\mathrm{M}}}-e_{s}^{p}=<\varepsilon_{x}>\cos ^{2} \alpha_{4}+<\varepsilon_{y}>\sin ^{2} \alpha_{4} ; \\
\left\langle\sigma_{x}\right\rangle=\frac{2 \sigma_{\mathrm{TM}}}{15\left(\cos ^{2} \alpha_{2}-\cos ^{2} \alpha_{1}\right)}\left[5\left(\cos ^{3} \alpha_{4}+\cos ^{3} \alpha_{3}\right)\left(\cos ^{2} \alpha_{2}-\cos ^{2} \alpha_{1}\right)-4\left(\cos _{2}{ }_{2}-\cos ^{5} \alpha_{1}\right)\right) \\
4\left(\cos ^{5} \alpha_{2}-\cos ^{5} \alpha_{1}\right)-20\left(\cos ^{3} \alpha_{2}-\cos ^{3} \alpha_{1}\right)-5\left(\cos ^{2} \alpha_{2}-\cos ^{2} \alpha_{1}\right) \times \\
\times\left(\cos ^{3} \alpha_{3}-\cos ^{3} \alpha_{4}\right)+15\left(\cos ^{2} \alpha_{2}-\cos ^{2} \alpha_{1}\right)\left(\cos _{3}-\cos _{4}\right)=0
\end{array}\right.
$$

Схема решения системы (26) может быть следующей. Задавая, например, в качестве параметра нагружения $\alpha_{4}$, из третьего, четвертого и пятого уравнений (26) имеем систему трех уравнений относительно трех неизвестных $\alpha_{1}, \alpha_{2}, \alpha_{3}$; зная величины всех углов $\alpha_{i}(\mathrm{i}=\overline{1,4})$, определяем величины $<\sigma_{\mathrm{x}}>,<\varepsilon_{\mathrm{x}}>,<\varepsilon_{\mathrm{y}}>$.Таким образом, схема деформирования одноосного образца вплоть до разрушения математически описана полностью.

Реализация изложенного алгоритма осуществлялась с использованием численных процедур решения нелинейных уравнений и систем нелинейных уравнений.

Экспериментальная проверка результатов расчета диаграмм упругопластического дефор-

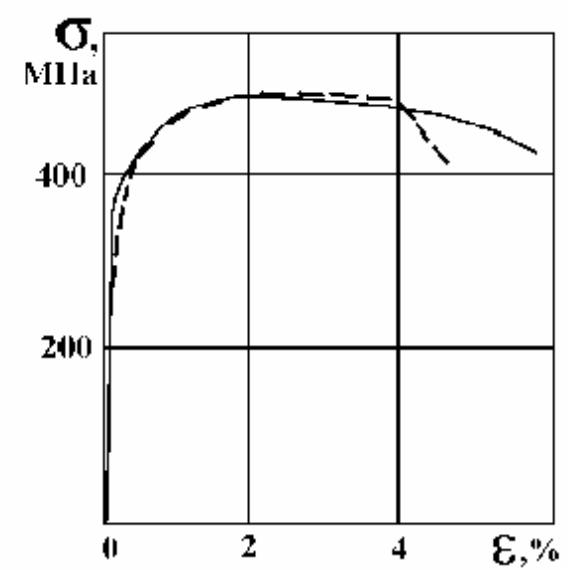

Р и с. 4. Экспериментальная (сплошная линия) и расчетная по структурной модели (штриховая линия) диаграммы упругопластического деформирования жаропрочного сплава ЭИ617 при Т $900{ }^{\circ} \mathrm{C}$

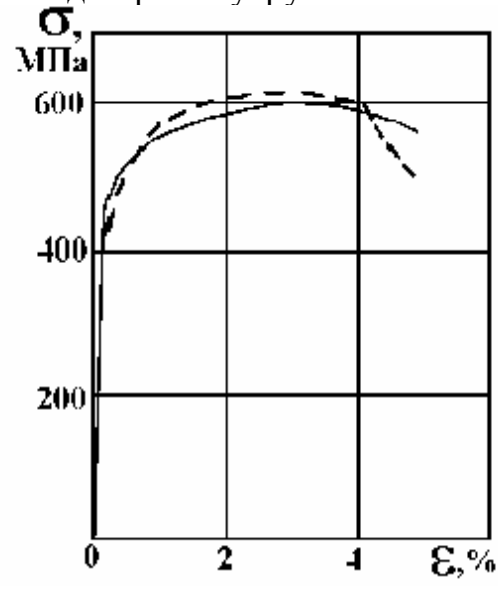

Р и с. 5. Экспериментальная (сплошная линия) и расчетная по структурной модели (штриховая линия) диаграммы упругопластического деформирования жаропрочного сплава ЭП693 при Т $850{ }^{\circ} \mathrm{C}$ 
мирования на основании структурной модели проводилась для различных классов материалов и температур. В качестве примера на рис. 4-5 приведены экспериментальные (сплошные линии) и расчетные (штриховые линии) по структурной модели диаграммы мгновенного упругопластического деформирования для жаропрочных сплавов ЭИ617 при $\mathrm{T}=900^{\circ} \mathrm{C}$ и ЭП693 при $\mathrm{T}=850^{\circ} \mathrm{C}$.

Экспериментальные данные для сплава ЭП693из работы [26], а для ЭИ617 взяты из работы [27].

На рис. 6 представлены расчетная (штриховая линия) и экспериментальная (сплошная линия) [28] диаграммы упругопластического деформирования при сжатии каменной соли при комнатной температуре.

Как следует из приведенных на рис. 4-6 графических зависимостей диаграмм упругопластического деформирования, для разных классов материлов наблюдается удовлетворительное соответствие расчетных и экспериментальных данных.

Проанализируем более подробно эффекты накопления поврежденности, следствием которых в конечном итоге является участок неустойчивого деформирования, с позиций предложенной струк-

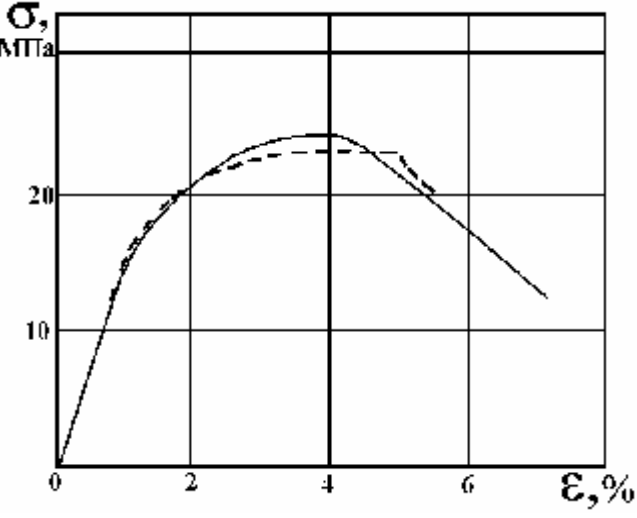

Р и с. 6. Экспериментальная сплошная линия [822] и расчетная по структурной модели (штриховая линия) диаграммы мгновенного упругопластического деформирования каменной соли при комнатной температуре при одноосном сжатии турной модели.

Идеализированные схемы 6,6 , 2 на рис.3 с феноменологических позиций описывают процесс накопления рассеянной поврежденности без эффекта существенного изменения эффективной площади сечения образца, так как здесь локальные элементы структурной модели не разрушаются. Однако в них накапливаются необратимые изменения, что ведет к концентрации энергии в наиболее нагруженных элементах системы. С феноменологических позиций эти схемы описывают концентрацию микронапряжений в областях, содержащих дефекты внутренней структуры материала (дефекты в геометрии атомной (кристаллической) решетки и межатомных связей, дислокации и т.п.). При этом нарушения сплошности материала на этих стадиях упрочнения не наблюдается.

Схемы $\partial$ и $е$ на рис. 3 моделируют ниспадающую ветвь диаграммы за счет разрушения локальных элементов модели и, как следствие этого, снижение несущей способности образца при растяжении. С феноменологических позиций эти схемы отражают процессы появления в материале микропор, микротрещин, пустот между кристаллами и т. п. Здесь происходит нарушение сплошности материала на микроуровне, что ведет на феноменологическом уровне к уменьшению эффективной площади поперечного сечения образца и снижению величины растягиваемой нагрузки при заданной скорости деформирования ( $<\dot{\varepsilon}>=$ const $)$.

Коснемся еще одного важного экспериментального факта, обнаруженного в работе [28], где экспериментально определялся модуль упругости горных пород в зависимости от степени накопления деформации и поврежденности. Отмечено, что модули упругости, определенные по продольным деформациям при разгрузке образца, изменяются незначительно в довольно широком диапазоне нагрузок: от начала нагрузки до максимально достижимого напряжения при сжатии $\sigma_{s}^{C}$ и в процессе разупрочнения образца до напряжения, составляющего около $60 \%$ $\sigma_{s}^{c}$, модули упругости практически были постоянными. При дальнейшем деформировании в области физического разрушения образца модули Юнга уменьшились в 2-3 раза. Параллельно с этим резко увеличились поперечные деформации по сравнению с продольными, так что это привело к увеличению объема образца примерно до 10 \% и более от первоначального. Все это свидетельствует о раскрытии микротрещин в материале на заключительной стадии деформирования, существенному разрушению микроструктуры образца и резкому уменьшению эффективной площади поперечного сечения образца.

Этот экспериментальный факт также моделируется структурной моделю и характеризует переход механизма разрушения от схемы $\partial$ к схеме $e$ на рис. 3. Здесь начинают разрушаться поперечно ориентированные локальные элементы, что ведет к резкой интенсификации поперечной макродеформации. С феноменологических позиций это, по всей видимости, соответствует появлению трещины и образованию шейки на образце. 
Таким образом, суммируя вышеизложенное, можно утверждать, что ниспадающий участок диаграммы деформирования является характеристикой материала, что подтверждено как экспериментальными, так и теоретическими исследованиями. Причиной этого участка является структурное разрушение микронеоднородной среды.

Здесь уместно отметить, что стремление описать накопление повреждений на закритической стадии деформирования породило развитие новой ветви в механике деформируемого твердого тела, а именно, разработку и усовершенствование моделей материала с целью описания накопления повреждений на закритической стадии деформирования. Это, в свою очередь, стимулировало развитие методов решения краевых задач пластичности по определению напряженно-деформированного состояния, предельной несущей способности и разрушению элементов конструкций, учитывающих возможность работы материала на стадии разрушения, которая характеризуется падающей ветвью диаграммы деформирования $[1,2,14,29,30]$.

На основании вышеизложенного можно сделать следующие выводы.

1. Предложенная структурная модель позволяет с позиций статики расчетным путем прогнозировать диаграмму упругопластического деформирования металлов, включая участок закритического деформирования.

2. Показано, что участок закритического деформирования связан с появлением и развитием зон микроразрушения локальных элементов, что с феноменологических позиций соответствует интенсивному накоплению поврежденности, уменьшению истинной площади поперечного сечения образца (пластическому разрыхлению материала) и одновременно уменьшению номинального напряжения при увеличении значения пластической деформации.

3. Предложенная модель может быть использована в качестве математического моделирующего комплекса упругопластического деформирования, накопления поврежденности и разрушения металлов с целью построения адекватных феноменологических моделей указанных процессов.

\section{БИБЛИОГРАФИЧЕСКИЙ СПИСОК}

1. Вильдеман В. Э., Соколкин Ю. В., Ташкинов А. А. Краевые задачи континуальной механики разрушения. Пермь, Препринт/УрОРАН, 1992. 78c.

2. Вильдеман В. Э., Соколкин Ю. В., Ташкинов А. А. Механика неупругого деформирования и разрушения композизионных материалов. М.: Наука, 1997.288c.

3. Фридман Я.Б. Механические свойства металлов. Ч.1 Деформация и разрушение. М.:Машиностроение, 1974. $472 \mathrm{c}$.

4. Лебедев А.А., Марусий О.И., Чаусов Н.Г., Зайцева Л.В. Исследование кинетики разрушения материалов на заключительной стадии деформирования // Проблемы прочности. 1982. №1. С.12-18.

5. Дубровина Г.И., Соковнин Ю.П., Гуськов Ю.П. и др. К теории накопления повреждений// Проблемы прочности. 1975. №2. C.21-24.

6. Пежина П. Моделирование закритического поведения и разрушения диссипативного твердого тела// Теоретические основы инженерных расчетов. 1984. Т.106. №4. С.107-117.

7. Фридман Я.Б. Механические свойства металлов. Ч.2. Механические испытания. Конструкционная прочность. М.: Машиностроение, 1974. 368c.

8. Черепанов Г.П. О закритических деформациях // Проблемы прочности. 1985. №8. С.3-8

9. Никитин Л.В., Рыжак Е.И. Об осуществимости состояний материала, соответствующих “падающему” участку диаграммы // Изв. АН. СССР. МТТ. 1986.№2. С.155-161.

10. Рыжак Е.И. К вопросу об осуществимости однородного закритического деформирования при испытаниях в жесткой трехосной машине // Изв. АН СССР. МТТ. 1991. №1. С. 111-127.

11. Лебедев А.А., Чаусов Н.Г. Установка для испытания материалов с построением полностью равновесных диаграмм деформирования // Проблемы прочности. 1981. №12. С.104-106.

12. Лебедев А.А., Чаусов Н.Г., Евечкий Ю.П. Методика построения полных диаграмм деформирования листовых материалов. // Проблемы прочности. 1986. №9. С.29-32.

13. Лебедев А.А., Чаусов Н.Г., Марусий О.И. и др. Кинематика разрушения листовой аустенитной стали на заключительной стадии деформирования. // Проблемы прочности. 1989. №3. С.16-21.

14. Стружаков В.В., Миронов В.И. Деформационное разрушение материала в элементах конструкций. Екатеринбург: Ур О РАН, 1995. 190 с.

15. Шин Р.Г., Катков В.Л. Механизмы деформирования микронеоднородной среды // Проблемы прочности. 1987. №10. C.72-74.

16. Работнов Ю.Н. О механизме длительного разрушения. // Вопросы прочности материалов и конструкций. Сб. тр. М.: Изд-во АН СССР, 1959. С.5-7.

17. Новожсилов В.В. О пластическом разрыхлении // Прикладная математика и механика. 1965. Т.29. №4. С. 681-689.

18. Качанов Л.М. О времени разрушения в условиях ползучести / Изв. АН СССР. ОТН. 1958. №8. С.26-31. 
19. Радченко В.П., Панферова Е.В. Структурная математическая модель упругопластического деформирования и разрушения металлов в одноосном случае // Вестн. СамГТУ. Вып. 4. Сер. «Физ. - мат. науки». Самара: СамГТУ, 1996. C.78-84.

20. Радченко В.П., Панферова Е.В. Моделирование неупругого деформирования и разрушения материалов на основании структурной модели // Численные и аналитические методы расчета конструкций: Тр. междунар. конф. Самара, 1998. С.82-86.

21. Новожилов В.В., Кадашевич Ю.И. Микронапряжения в конструкционных материалах. Л.: Машиностроение. Ленингр. отд-ние), 1990. 223 с.

22. Гохфельд Д.А., Садаков О.С. Пластичность и ползучесть элементов конструкций при повторном нагружении. М.: Машиностроение, 1984. $256 \mathrm{c}$.

23. Розенберг B.M. Основы жаропрочности металлических материалов. М.: Металлургия, 1973. 328c.

24. Weng G.J. Aplisically consistent method for the predictions of creep behavior of metals // Trans. ASME. J.Appl. Mech. 1979. №4. P.800 - 804.

25. Besseling J.F. Plasticity and creep theory in engineering mechanics // Top. Appl. Continuum. Mech.- Wien - New York. 1974. P. $115-135$.

26. Радченко В.П. Энергетический вариант одноосной теории ползучести и длительной прочности // Журн. прикл. мех. и техн. физики. 1991. №4. С. $172-179$.

27. Бульгин и др. Атлас диаграмм растяжения при высоких температурах, кривые ползучести и длительной прочности сталей и сплавов для двигателей. М.: Оборонгиз, 1953. 173 с.

28. Карташов Ю.М. , Матвеев Б.В., Михеев Г.В., Фадеев А.Б. Прочность и деформируемость горных пород. М.: Недра, 1979. $269 \mathrm{c.}$

29. Волков С.Д. Функция сопротивления материалов и постановка краевых задач механики разрушения // УНЦ АН СССР. Ин - т металлургии. Препринт. Свердловск, 1986. 65с.

30. Ибрагимов B.A., Клюшников В.Д. Некоторые задачи для сред с падающей диаграммой // Изв. АН СССР. МТТ. 1971. №4. C.116 - 121. 